\title{
Numerical analysis for cracked functionally graded materials by
}

\section{finite block method}

\author{
J. Li ${ }^{1, a}$, C. Shi ${ }^{2, b}$, T. Alexander ${ }^{3, c}$ and P.H. Wen ${ }^{2, d}$ \\ ${ }^{1}$ School of Mathematics and Statistics, Changsha University of Science and Technology, China \\ ${ }^{2}$ School of Engineering and Materials Science, Queen Mary University of London, London, UK \\ ${ }^{3}$ Parks College of Engineering, Aviation and Technology, Saint Louis University, USA \\ a lijingnew@126.com, ${ }^{\mathrm{b}}$ c.shi@qmul.ac.uk, ${ }^{\mathrm{c}}$ theodosios.alexander@googlemail.com, \\ dp.h.wen@qmul.ac.uk
}

Keywords: functionally graded materials, finite block method, stress intensity factors

\begin{abstract}
The finite block method (FBM) is developed to determine stress intensity factors with orthotropic functionally graded materials under static and dynamic loads in this paper. The higher order derivative matrix for two and three dimensional problems can be constructed directly. For linear elastic fracture mechanics, the COD and $J$-integral techniques to determine the stress intensity factors are applied. Several examples are given and comparisons have been made with both analytical solutions and the finite element method in order to demonstrate the accuracy and convergence of the finite block method.
\end{abstract}

\section{Introduction}

To deal with physically realistic problems, such as transient heat conduction in anisotropic and non- homogeneous media, is a complex task and the mathematical modelling becomes very complicated for both analytical and numerical analysis. There are two categories in the numerical engineering, i.e. the domain-type and boundary-type methods. Domain-type methods including the finite element method (FEM) and the boundary-type methods the boundary element method (BEM) can be found in the numerical engineering (see Kim and Paulino [1], Aliabadi [2]). Although the BEM is one of the accurate and efficient methods, the fundamental solutions or Green's functions are required. For FGM, the governing equations contain many coefficients related to the material properties which are dependent on the coordinates and directions of fibre in composites, see Sladek et al [3]. The Finite Block Method, based on the point collocation method was developed firstly to solve the heat conduction problem in the functionally graded media and anisotropic materials by Li and Wen [4]. The essential feature of the FBM is that the physical domain is divided into few blocks and for each block the partial differential matrices are obtained in terms of nodal values using mapping technique. For linear elastic fracture mechanics, the static stress intensity factors are evaluated by both crack opening displacement (COD) and $J$-integral technique for both isotropic and orthotropic FGMs. To demonstrate the accuracy and efficiency of the FBM, several numerical examples are given with ccomparisons made with the finite element method and local PetrovGalerkin approach.

\section{Finite block method with FGMs}

Assumed that the material properties are dependent on the spatial coordinates in a nonhomogeneous material. The relationship between stress and strain anisotropic materials gives

$$
\left[\begin{array}{c}
\varepsilon_{11} \\
\varepsilon_{22} \\
2 \varepsilon_{12}
\end{array}\right]=\left[\begin{array}{lll}
\beta_{11} & \beta_{12} & \beta_{16} \\
\beta_{12} & \beta_{22} & \beta_{26} \\
\beta_{16} & \beta_{26} & \beta_{66}
\end{array}\right]\left[\begin{array}{c}
\sigma_{11} \\
\sigma_{22} \\
\sigma_{12}
\end{array}\right]
$$

where $\beta_{i j}$ are the elastic compliances of the FGMs. The compliance coefficients can be written in terms of the engineering constants as 
$\beta_{11}=1 / E_{1}, \beta_{22}=1 / E_{2}, \beta_{12}=-v_{12} / E_{1}=-v_{21} / E_{2}$,

$\beta_{16}=\eta_{12,1} / E_{1}=\eta_{1,12} G_{12}, \beta_{26}=\eta_{12,2} / E_{2}=\eta_{2,12} G_{12}, \beta_{66}=1 / G_{12}$

where $E_{1}$ and $E_{2}$ are the Young's moduli along two axes of coordinate, $v_{12}$ and $v_{21}$ are Poisson's ratios, $G_{12}$ is the shear modulus and $\eta_{j k, l}$ and $\eta_{l, j k}$ are the mutual coefficients of first and second. The inverse form of the relationship in (1) yields

$\left[\begin{array}{c}\sigma_{11} \\ \sigma_{22} \\ \sigma_{12}\end{array}\right]=\left[\begin{array}{lll}Q_{11} & Q_{12} & Q_{16} \\ Q_{12} & Q_{22} & Q_{26} \\ Q_{16} & Q_{26} & Q_{66}\end{array}\right]\left[\begin{array}{c}\varepsilon_{11} \\ \varepsilon_{22} \\ 2 \varepsilon_{12}\end{array}\right]$.

For plane stress orthotropic elasticity, material mechanical constants give

$Q_{11}=\frac{E_{1}}{1-v_{12} v_{21}}, Q_{12}=\frac{v_{12} E_{1}}{1-v_{12} v_{21}}, Q_{22}=\frac{E_{2}}{1-v_{12} v_{21}}, Q_{16}=Q_{26}=0, Q_{66}=G_{12}$.

The equilibrium equations give

$$
\sigma_{\alpha \beta, \beta}+b_{\alpha}=0 \quad \alpha, \beta=1,2, \quad \mathbf{x} \in \Omega
$$

where $b_{\alpha}$ are body forces. Applying the differential matrices over (5) for each block, and substituting (3) into equilibrium equation in (5) results, in matrix form [4], as

$\left(\mathbf{D}_{1} \mathbf{Q}_{11} \mathbf{D}_{1}+\mathbf{D}_{2} \mathbf{Q}_{66} \mathbf{D}_{2}\right) \mathbf{u}_{1}+\left(\mathbf{D}_{1} \mathbf{Q}_{12} \mathbf{D}_{2}+\mathbf{D}_{2} \mathbf{Q}_{66} \mathbf{D}_{1}\right) \mathbf{u}_{2}+\mathbf{b}_{1}=\mathbf{0}$

$\left(\mathbf{D}_{2} \mathbf{Q}_{12} \mathbf{D}_{1}+\mathbf{D}_{1} \mathbf{Q}_{66} \mathbf{D}_{2}\right) \mathbf{u}_{1}+\left(\mathbf{D}_{1} \mathbf{Q}_{66} \mathbf{D}_{1}+\mathbf{D}_{2} \mathbf{Q}_{22} \mathbf{D}_{2}\right) \mathbf{u}_{2}+\mathbf{b}_{2}=\mathbf{0}$

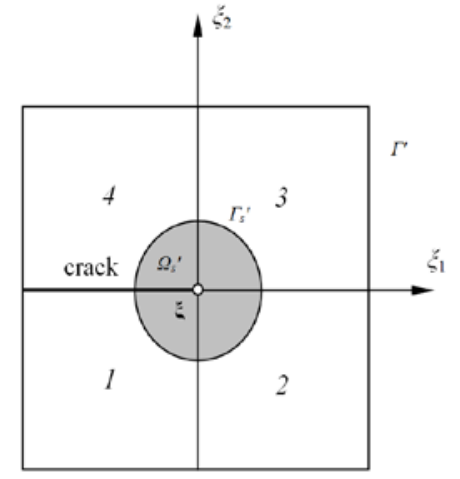

(a)

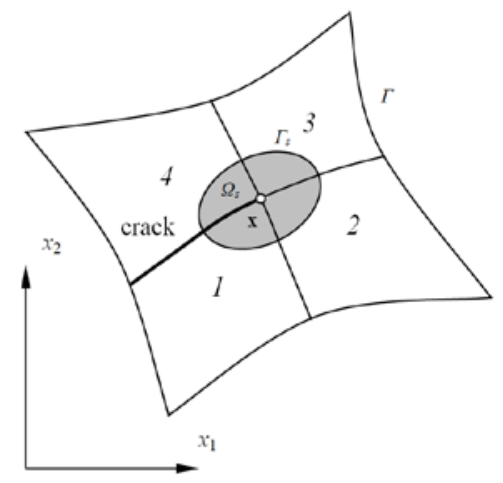

(b)

Figure 1. Four blocks and integral domain.

where $\mathbf{u}_{\alpha}=\left\{u_{\alpha}^{(1)}, u_{\alpha}^{(2)}, \ldots, u_{\alpha}^{(M)}\right\}^{T}$, $\mathbf{b}_{\alpha}=\left\{b_{\alpha}^{(1)}, b_{\alpha}^{(2)}, \ldots, b_{\alpha}^{(M)}\right\}^{T}$ are nodal value vectors and

$\mathbf{Q}_{i j}=\left(\begin{array}{cccc}Q_{i j}^{(1)} & 0 & \ldots & 0 \\ 0 & Q_{i j}^{(2)} & \ldots & 0 \\ \ldots & \ldots & \ldots & \ldots \\ 0 & 0 & \ldots & Q_{i j}^{(M)}\end{array}\right)$.

in which $Q_{i j}^{(k)}$ indicates the elasticity coefficient at node $k$. The boundary conditions give

$u_{\alpha}(\mathbf{x})=u_{\alpha}^{0}(\mathbf{x}) \quad \mathbf{x} \in \Gamma_{u}, \quad \sigma_{\alpha \beta} n_{\beta}=t_{\alpha}^{0}(\mathbf{x}) \quad \mathbf{x} \in \Gamma_{q}$

where $u_{\alpha}^{0}$ and $t_{\alpha}^{0}$ are specified displacements and tractions on the boundary. Obviously there are $2 M$ linear algebraic equations from (6) and (8) for each block, and therefore, all nodal values of displacement should be determined. In the case of more than one blocks, the continuous condition on the interface between blocks I and II gives

$u_{\alpha}^{I}(\mathbf{x})-u_{\alpha}^{I I}(\mathbf{x})=0, t_{\alpha}^{I}(\mathbf{x})+t_{\alpha}^{I I}(\mathbf{x})=0 . \quad \mathbf{x} \in \Gamma_{\mathrm{int}}$

\section{Stress intensity factors by $\mathrm{J}$-integral technique}

Consider the gradient for strain energy [1]

$\frac{\partial W}{\partial x_{\eta}}=\frac{\partial W}{\partial \varepsilon_{\alpha \beta}} \frac{\partial \varepsilon_{\alpha \beta}}{\partial x_{\eta}}+\frac{\partial W}{\partial Q_{\alpha \beta}} \frac{\partial Q_{\alpha \beta}}{\partial x_{\eta}}$

where $W$ is strain energy density and defined as 


$$
W\left(\varepsilon_{\alpha \beta}, Q_{\alpha \beta}\right)=\frac{1}{2} \sigma_{\alpha \beta} \varepsilon_{\alpha \beta}, \quad \sigma_{\alpha \beta}=\frac{\partial W}{\partial \varepsilon_{\alpha \beta}}
$$

The equilibrium equations can be expressed as

$$
\left(W \delta_{\beta \eta}-\sigma_{\alpha \beta} u_{\alpha, \eta}\right)_{, \beta}-\frac{\partial W}{\partial Q_{\alpha \beta}} \frac{\partial Q_{\alpha \beta}}{\partial x_{\eta}}=0
$$

where $\delta_{\beta k}$ is the Kronecker delta. A closed curve $\Gamma$ in the local coordinate is introduced along with the domain $\Omega$ which it encloses. Integrating (12) over the domain $\Omega$ with the divergence theorem gives

$$
\oint\left[W n_{\eta}-T_{\alpha} u_{\alpha, \eta}\right] d \Gamma-\int_{\Omega}\left(\frac{\partial W}{\partial Q_{\alpha \beta}} \frac{\partial Q_{\alpha \beta}}{\partial x_{\eta}}\right) d \Omega=0, \quad \eta=1,2
$$

where $T_{\alpha}=\sigma_{\alpha \beta} n_{\beta}$ is traction, $n_{\beta}$ is the components of the outward unit normal vector to $\Gamma$. Since the stress and strain fields are singular at a crack tip with $1 / \sqrt{r}$, then the $J$-integral, which is independent of integral path, become

$$
J=\int_{\Gamma_{0}}\left[W n_{1}-T_{\alpha} u_{\alpha, 1}\right] d \Gamma-\int_{\Omega_{0}}\left(\frac{\partial W}{\partial Q_{\alpha \beta}} \frac{\partial Q_{\alpha \beta}}{\partial x_{1}}\right) d \Omega
$$

for traction free of crack surfaces when $\eta=1$, and by linear elastic fracture mechanics

$$
J=\frac{K_{I}^{2}+K_{I I}^{2}}{E_{\text {tip }}}
$$

and $\Gamma_{0}=\Gamma-\Gamma_{c}^{+}-\Gamma_{c}^{-}$for isotropic FGMs, $E_{\text {tip }}$ is Young's modulus at crack tip in plane stress case. For linear elasticity, the domain integration in (14) is bounded and is evaluated by any standard integral techniques. From the relationship of COD, the ratio of stress intensity factors is proximated

$$
\varphi=\frac{K_{\mathrm{II}}}{K_{\mathrm{I}}}=\frac{\Delta u}{\Delta v}
$$

when $r=r_{0}$, then the stress intensity factors are obtained

$$
K_{I}=\sqrt{\frac{E_{\mathrm{tip}} J}{1+\varphi^{2}}} \operatorname{sign}(\Delta v), K_{I I}=\sqrt{\frac{E_{\mathrm{tip}} J}{1+\varphi^{2}}}|\varphi| \operatorname{sign}(\Delta u)
$$

where $r_{0}$ is distance between selected point and crack tip. In general case, crack tip is surrounded by four blocks as shown in Figure 1. For the convenience of computation, the integral domain is selected as a circle of radius $R$ centred at crack tip in the normalized domain. Therefore, the coordinate of local integral boundary $S^{\prime}\left(\xi_{1 s}, \xi_{2 s}\right)$ in the mapping domain is

$\xi_{1}=\xi_{1 l}^{0}+R \cos \theta, \xi_{2}=\xi_{2 l}^{0}+R \sin \theta$

where $\left(\xi_{1 l}^{0}, \xi_{2 l}^{0}\right)$ is coordinate of crack tip in mapping domain, the coordinate $S\left(x_{1}, x_{2}\right)$

$$
x_{\alpha l}=\sum_{k=1}^{8} N_{k}\left(\xi_{1}, \xi_{2}\right) x_{\alpha l}^{(k)}
$$

where $\left(x_{1 l}^{(k)}, x_{2 l}^{(k)}\right)$ denotes the coordinate of seed $k$ in the real for block $l$ domain surrounding the crack tip. The $J$-integral can be written, in the normalized domain, as

$$
J=\sum_{l=1}^{4}\left[\int_{\theta_{1}^{(l)}}^{\theta_{2}^{(l)}}\left[W n_{1}-T_{\alpha} u_{\alpha, 1}\right] \sqrt{f_{1 l}^{2}+f_{2 l}^{2}} R d \theta-\int_{\theta_{1}^{(l)}}^{\theta_{2}^{(l)}} \int_{0}^{R}\left(\frac{\partial W}{\partial Q_{\alpha \beta}} \frac{\partial Q_{\alpha \beta}}{\partial x_{1}}\right)\left|J_{l}\right| \rho d \rho d \theta\right]
$$

\section{Example}

An isotropic FGM finite two-dimensional strip with an edge crack of length $a$ shown in Figure 2 is considered. Tensile uniform load is applied on top of the plate and the bottom is fixed. Young's 
modulus is an exponential function of $x_{1}$ only, i.e. $E_{1}=E_{2}=E_{0} \exp \left(\alpha x_{1} / w\right)$ for two directions, in which $\alpha=\ln \left(E_{w} / E_{0}\right), E_{0}$ and $E_{w}$ are Young's moduli on the left-hand side and right-hand side of the strip respectively. The Poisson ratio $v_{12}=v_{21}=v=0.3$ and shear modulus is assumed as $G_{12}=E_{1} / 2(1+v)$. Plane stress is considered. In this modelling, four blocks are used and the nodal with $N_{1}=N_{2}=21$ for each and dimensions $w=2 a$ and $h=2 w$. The stress intensity factors under the tensile and bending loads are presented in Table 1 for different ratio $E_{w} / E_{0}$.

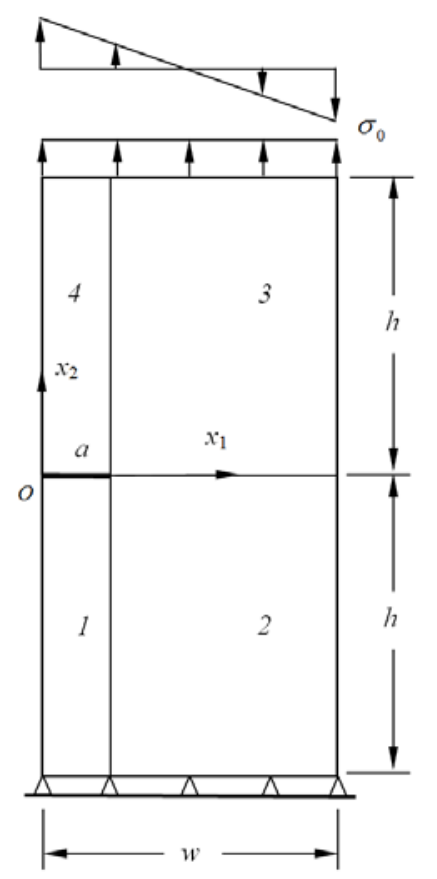

Table 1. Normalized SIFs of edge cracked plate $K_{I} / \sigma_{0} \sqrt{\pi a}$

\begin{tabular}{|c|c|c|c|c|c|}
\hline & $E_{w} / E_{0}$ & COD & J-int & Erdogan $^{[5]}$ & $\operatorname{Kim}^{[1]}$ \\
\hline \multirow{5}{*}{  } & 0.1 & 3.4879 & 3.4674 & 3.570 & 3.496 \\
\hline & 0.2 & 3.3224 & 3.2806 & 3.326 & 3.292 \\
\hline & 1 & 2.9026 & 2.8220 & NA & 2.822 \\
\hline & 5 & 2.4684 & 2.3627 & 2.365 & 2.366 \\
\hline & 10 & 2.2826 & 2.1704 & 2.223 & 2.175 \\
\hline \multirow{5}{*}{ 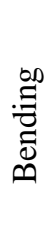 } & 0.1 & 2.2669 & 2.1227 & 2.215 & 2.145 \\
\hline & 0.2 & 2.0408 & 1.9145 & 1.953 & 1.925 \\
\hline & 1 & 1.5918 & 1.4942 & NA & 1.496 \\
\hline & 5 & 1.2366 & 1.1561 & 1.151 & 1.158 \\
\hline & 10 & 1.1066 & 1.0317 & 1.035 & 1.035 \\
\hline
\end{tabular}

Figure 2 Edge cracked plate.

\section{Summary}

The essential features of the proposed numerical techniques in this paper can be summarised as:

(1) The physical domain is divided into several blocks with four edges and eight seeds for each blocks. The ratio of two sides for each block satisfies $1 / 8<l_{1} / l_{2}<8$;

(2) The distribution of node in the normalised domain is chosen to be the Chebyshev's roots for crack problems in order to obtained higher accurate results;

(3) Anisotropic functionally graded materials with all kinds of boundary value problems can be formulated and solved easily.

\section{Acknowledgement}

The first author would like to thank the support partially from the Natural and Science Foundation Council of China (No. 11301040).

\section{References}

[1] J.H. Kim, G.H. Paulino: Int J Numer Meth Eng 53 (2002), 1903-35.

[2] Aliabadi MH. The Boundary Element, Applications in Solids and Structures. John Wiley and Sons, Ltd., Chichester, England; 2002.

[3] J. Sladek, V.Sladek, Ch.Zhang: Engng Anal. with Bound. Ele. 29 (2005), 597-609

[4] P.H. Wen, P. Cao and T. Korakianitis: Engng Anal. with Bound. Ele. 46 (2014), 116-125.

[5] F. Erdogan, B.H. Wu: ASME Journal of Applied Mechanics 64 (1997), 449-456. 